started in 2010. Patients from the age of 13 who were questioned about their health behavior and followed up for at least 2 years were selected. HRB were quantified and compared with those of subjects from the general population after matching for age and sex. Data from 2-year follow-up (FU) were used to analyze correlates of multiple risk behavior defined as involvement in two or more risky behaviors.

Results: A total of 209 adolescents with JIA (63\% female, mean age at baseline $14.4 \pm 0.9$, mean disease duration $2.6 \pm 2.0)$ and 138 healthy peers $(55 \%$ female, mean age $14.5 \pm 1.0$ ) were included. At baseline, $51 \%$ of patients were treated with a DMARD, $21 \%$ with a biologic (FU: $59 \%$ and $38 \%$ ). The most common JIA category was rheumatoid factor negative polyarthritis $(28 \%)$. While at baseline $20 \%$ of patients and $4 \%$ of controls did not engage in regular physical activity, the proportion at follow-up amounted to $16 \%$ and $10 \%$, respectively (OR 3.69; $95 \% \mathrm{Cl}: 1.01-13.50)$. In both groups the proportion of regular smokers, alcohol consumers and drug users increased during the observation period. Significant group differences were found in terms of alcohol consumption and smoking habits, but not in relation to illicit and legal drugs (see table). Patients stated significantly more often that they had not used a condom during their last sexual intercourse $(28 \%$ vs. $19 \%$ controls, $\mathrm{p}<0.05)$. Multiple risk behavior was associated with PedsQL ${ }^{\mathrm{TM}}$ total score (OR 0.96; 95\% Cl: 0.92-0.99) and disease duration (OR 0.75; 95\%Cl: 0.57-0.98).

\begin{tabular}{lcccc}
\hline Forms of HRB & \multicolumn{2}{c}{ Baseline } & \multicolumn{2}{c}{ 2-year follow-up } \\
\hline & JIA & CG & JIA & CG \\
& $\mathrm{N}=209(\%)$ & $\mathrm{N}=138(\%)$ & $\mathrm{N}=209(\%)$ & $\mathrm{N}=138(\%)$ \\
& & & & \\
Inactivity/smoking/alcohol consumption & 20.3 & 3.7 & 16.2 & 9.5 \\
$\quad$ Physical activity <1/week & 58.9 & 43.4 & 54.4 & 42.3 \\
$\quad$ Active $\leq 2 /$ week & 2.4 & 0.7 & 7.8 & 2.2 \\
$\quad$ Regular smokers & 3.8 & 3.6 & 15.8 & 23.2 \\
$\quad$ Regular alcohol consumers & 1.9 & 1.4 & 8.1 & 8.0 \\
Drug use & 0.5 & 0.0 & 4.8 & 3.6 \\
$\quad$ 12-month prevalence & 3.3 & 1.4 & 25.2 & 23.2 \\
$\quad$ Multiple consumers & 28.6 & 50.0 & 28.0 & 19.4 \\
Sexual intercourse & & & &
\end{tabular}

Conclusion: Although adolescents with JIA became more physically active during the course of the disease, they are as likely, or more likely, to take risky behaviors than their healthy peers, except for alcohol consumption. In order to achieve optimal outcomes, addressing emotional wellbeing and providing mandatory anticipatory guidance appears to be warranted in this population.

References:

[1] Villarreal-Rodriguez D et al. Adolescents with chronic disease and participation in risky behaviors. Medicina Universitaria 2013;15(58):21-25.

Acknowledgments: ICON is supported by a grant from the German Federal Ministry of Education and Research (FKZ 01 ER 1504A)

Disclosure of Interests: Florian Milatz: None declared, Ina Liedmann: None declared, Martina Niewerth: None declared, Jens Klotsche: None declared, Gerd Horneff Grant/research support from: AbbVie, Chugai, Merck Sharp \& Dohme, Novartis, Pfizer, Roche, Speakers bureau: AbbVie, Bayer, Chugai, Merck Sharp \& Dohme, Novartis, Pfizer, Roche, Frank Weller-Heinemann: None declared, Ivan Foeldvari Consultant of: Novartis, Daniel Windschall: None declared, Kirsten Minden Consultant of: GlaxoSmithKline, Sanofi, Speakers bureau: Roche

DOI: 10.1136/annrheumdis-2020-eular.2155

\section{THU0513 MEDICATION BURDEN IN YOUNG ADULTS WITH JUVENILE IDIOPATHIC ARTHRITIS - RESULTS OF AN OBSERVATIONAL COHORT STUDY}

L. Montag ${ }^{1,2}$, J. Klotsche ${ }^{2}$, M. Niewerth ${ }^{2}$, S. Tatsis ${ }^{3}$, E. Seipelt ${ }^{4}$, P. Hoff ${ }^{5}$, G. Horneff ${ }^{6}$, K. Minden ${ }^{1,2} .{ }^{1}$ Charité Universitätsmedizin Berlin, Berlin, Germany; ${ }^{2}$ German Rheumatism Research Centre, Berlin, Germany; ${ }^{3}$ Marienkrankenhaus, Hamburg, Germany; ${ }^{4}$ Immanuel Krankenhaus Berlin, Berlin, Germany;

${ }^{5}$ Endokrinologikum Berlin, Berlin, Germany; ${ }^{6}$ Asklepios Kinderklinik Sankt Augustin, Sankt Augustin, Germany

Background: Juvenile idiopathic arthritis (JIA) often persists into adulthood, and many young adults with JIA still need antirheumatic medication. Rheumatologists prescribe DMARDs and glucocorticoids, but are not always aware of other medications taken by patients concurrently. Self-reported medication use was used to obtain information on drug exposure of young adults with JIA.

Objectives: To evaluate medication use in a large cohort of young adults with JIA ever treated with DMARDs.
Methods: Patients ever treated with DMARDs and prospectively observed in the JIA biologic registry JuMBO were asked about their drug consumption at each JuMBO visit. In addition, patients reported their current health status in terms of disease activity and pain (scored on numerical rating scales 0-10), functional ability (by HAQ) and quality of life (by SF-36). The Anatomical Therapeutic Chemical Classification System for medicinal products (ATC) was used to classify self-reported medication use. Local therapies, with the exception of ophthalmological drugs, and cough and cold remedies were not included

Results: A total of 1,306 young adults ( $68 \%$ female) with JIA and a mean disease duration of $13.6 \pm 6$ years (ys) were included in the analysis. The majority of them were classified as polyarticular-onset JIA (35.6\%), 20.5\% as enthesitis-related arthritis.

At the last follow-up (FU), the patients' mean age was $23.1 \pm 4.1$ ys. They had received a mean of $2.6 \pm 1.4$ DMARDs, $79 \%$ were ever treated with biologics. At FU, patients used on average $1.9 \pm 1.8$ drugs. About one in five patients (296 $22.7 \%)$ reported no medication use at all, $367(28.1 \%)$ reported only DMARD use. The most frequently reported drugs were DMARDs (64\%), NSAIDs (48\%), glucocorticoids (19\%), followed by analgesics (10.6\%), drugs for acid-related disorders $(6.9 \%)$ and anti-infectives for systemic use $(6.1 \%)$. Antidepressant drug use reported $3.4 \%$ and antihypertensive drug use $3.1 \%$ of the patients Women used significantly more frequently NSAIDs, glucocorticoids, non-opioid analgesics and thyroid medication. There were 178 (14\%) patients who received at least 3 other medications in addition to DMARDs. This patient group frequently reported the use of pain medication (74\% NSAIDs, $23 \%$ non-opioid analgesics $20 \%$ opioid drugs) and antidepressants (16\%) and had been treated late with bDMARDs ( $7.4 \pm 4.9$ ys after symptom onset). There were significant differences in drug usage between patients with various JIA categories (table). Moreover, the use of glucocorticoids, antihypertensives and antidepressants in adulthood (adjusted by propensity scores) increased with longer time from symptom onset to bDMARD start.

Table. Self-reported medication use in patients with different JIA categories at the last JuMBO FU

Sys JIA OA, OA, ext.RF- PARF+ PA ERA PsA Other P pers. arthritis value

$\mathrm{N}=66 \mathrm{~N}=117 \mathrm{~N}=227 \mathrm{~N}=350 \quad \mathrm{~N}=115 \mathrm{~N}=268 \mathrm{~N}=116 \quad \mathrm{~N}=47$

Drugs per patient, mean $2.7 \pm 2.21 .3 \pm 1.62 .2 \pm 2.01 .8 \pm 1.82 .7 \pm 2.01 .5 \pm 1.51 .9 \pm 1.71 .7 \pm 1.8<0.00$ Drugs, in \%

DMARDs

bDMARDs

SMARD

Glucocorticoids

GSAIDs

Drugs for acid related disorders

Analgesics

Opioids

Antiinfectives

Antidepressants

Antihypertensives

Antithrombotics

$\begin{array}{ccccccccc}68 & 45 & 74 & 60 & 87 & 60 & 62 & 57 & <0.001 \\ 59 & 32 & 64 & 48 & 73 & 53 & 53 & 47 & <0.001 \\ 27 & 25 & 34 & 28 & 46 & 21 & 29 & 23 & <0.001 \\ 32 & 10 & 23 & 19 & 35 & 10 & 19 & 17 & <0.001 \\ 36 & 36 & 52 & 48 & 55 & 44 & 55 & 55 & 0.008 \\ 6 & 3 & 8 & 7 & 7 & 6 & 8 & 15 & 0.314 \\ & & & & & & & & \\ 12 & 6 & 11 & 13 & 14 & 8 & 10 & 11 & 0.198 \\ 9 & 2 & 4 & 4 & 4 & 2 & 2 & 4 & 0.181 \\ 9 & 4 & 7 & 7 & 3 & 6 & 7 & 6 & 0.641 \\ 6 & 3 & 5 & 3 & 1 & 5 & 3 & 0 & 0.319 \\ 11 & 1 & 3 & 3 & 5 & 2 & 3 & 6 & 0.005 \\ 5 & 1 & 1 & 1 & 4 & 0.4 & 0 & 0 & 0.027\end{array}$

Conclusion: Self-reported medication use adds important information when assessing the long-term outcome of JIA. About $15 \%$ of JIA patients ever exposed to DMARDs, especially those with late start of bDMARD therapy, have a high medication or disease burden in young adulthood.

Acknowledgments: JUMBO - joint unconditional grant from Pfizer, Abbvie, Roche

Disclosure of Interests: Laura Montag: None declared, Jens Klotsche: None declared, Martina Niewerth: None declared, Stefanie Tatsis: None declared, Eva Seipelt: None declared, Paula Hoff Consultant of: BMS, Amgen, Speakers bureau: Novartis, Jansen, Pfizer, Mylan, BMS, Gerd Horneff Grant/research support from: AbbVie, Chugai, Merck Sharp \& Dohme, Novartis, Pfizer, Roche, Speakers bureau: AbbVie, Bayer, Chugai, Merck Sharp \& Dohme, Novartis Pfizer, Roche, Kirsten Minden Consultant of: GlaxoSmithKline, Sanofi, Speakers bureau: Roche

DOI: 10.1136/annrheumdis-2020-eular.1894

\section{THU0514 \\ BIOLOGICAL THERAPY IN JUVENILE PSORIATIC ARTHRITIS - 15 YEARS OF SINGLE CENTER EXPERIENCE}

I. Nikishina ${ }^{1}$, S. Arsenyeva ${ }^{1}$, M. Kaleda ${ }^{1}$, A. Shapovalenko ${ }^{1}$, O. Kostareva ${ }^{1}$, A. Latypova ${ }^{1}$, T. Pachkoria ${ }^{1} .{ }^{1}$ V. A. Nasonova Research Institute of Rheumatology, Paediatric, Moscow, Russian Federation 
Background: Juvenile psoriatic arthritis (JPsA) is one of the clinical variants of juvenile idiopathic arthritis (JIA), which is often characterized by an unfavorable course, refractory to therapy, requiring the prescription of Biological agents (BA).

Objectives: analysis of BA use in patients with JPsA and therapy survival, switching to another line of BA.

Methods: the retrospective cohort study included 1095 JIA patients who received BA and were observed in our clinic from 2004 to 2019. All cases of new onset psoriasis were collected; clinical features of disease onset and course, exposure to Methotrexate (MTX) and BA, presence of ANA, HLA B27 were studied.

Results: among 1095 JIA patients who received BA over the past 15 years, a separate cohort of patients with JPsA for analysis was allocated. We identified 50 pts (57\% female) aged 2-18 years (Me 13.3) at the time of initiation of therapy. All patients met the JPsA classification criteria, the average age of arthritis onset was $7.4 \pm 5.3$ years (ME 6.75). However, cutaneous psoriasis occurred only in $68 \%$ (34 pts), with manifestation at the age of $10 \pm 5$ years. In 25 of 34 pts $(73.5 \%)$ the development of psoriasis was preceded by joint manifestations in an average of $5 \pm 3.9$ (ME 3 ) years. 6 pts from $1095(0.65 \%)$ developed psoriasis under BA therapy: infliximab - 2 cases (0.62/100PY), adalimumab - 3 (0.15/100PY), abatacept - 1 (0.31/100PY). 2/6 pts was ANA+, 3/6 - HLA B27+ Average age of disease onset was $9.8 \pm 7.8$ years; BA exposure before psoriasis was 2.7 \pm 1.1 years and in 3.6 \pm 1.3 (ME 4) after the onset of arthritis. Therapy was continued in $4 / 6$ pts; switched from infliximab to adalimumab in 2 . Serious comorbid pathology was associated with JPsA in 7 pts (type 1 diabetes mellitus - 2 pts; Down syndrome; endogenous mental illness (schizophrenia); oligophrenia; ovaries polycystic; acute lymphoblastic leukemia in a state of incomplete remission). The clinical picture of the disease was represented by polyarthritis in $84 \%$, oligoarthritis in $8 \%$, the same number of patients $8 \%$ had an axial lesion. Sacroiliitis was detected in 20 patients (40\%), dactylitis in 21 (42\%), and uveitis in $10(20 \%)$. HLA B27 was detected in 16/35 pts (45\%), 32\% pts were ANA-positive. The duration of the disease at the time of application of the first BA was $5 \pm 4$ (Me 3.75) years. In 49 patients, BA was used in combination with methotrexate. The total number of BA courses switching included was 80 (infliximab-19, adalimumab-22, etanercept-27, golimumab-4, abatacept-5, tocilizumab-2, rituximab-1). $49 \%$ of patients have experience of using $>2$ BA (16 pts-2 BA, 4 pts-3 BA, 1 pt - 5 BA). Primary/secondary inefficiency (18/35; $51 \%)$, adverse events $(8 / 35 ; 23 \%)$, organizational difficulties in market access mostly after the age of $18(7 / 35 ; 20 \%)$, and remission $(2 / 35 ; 6 \%)$ were the reasons for the withdrawn of BA. Among the serious adverse events, multiple sclerosis was registered after 6 years of abatacept using (the relationship with the drug used has not been proven), pregnancy in the 3rd year of adalimumab use (interruption at 16 weeks); serious local reaction after etanercept using- 1; infusion reactions (1-rituximab, 2-infliximab); uveitis de novo (2- etanercept).

Conclusion: JPsA is one of the most severe variants of JIA, characterized by a high proportion of serious comorbid conditions, the development of refractoriness and adverse events of BA therapy (including uveitis, multiple sclerosis), requiring switching to line 2 and 3 with a limited choice of BA with pediatric indications. Special study requires the manifestation of psoriasis de novo mainly developed during TNF-monoclonal antibodies therapy.

Disclosure of Interests: None declared

DOI: 10.1136/annrheumdis-2020-eular.6366

\section{THU0515 AND ADOLESCENCE - UPDATE FROM THE NATIONAL PEDIATRIC RHEUMATOLOGY DATABASE}

C. Sengler ${ }^{1}$, M. Niewerth ${ }^{1}$, N. Geisemeyer ${ }^{1}$, H. Girschick ${ }^{2}$, A. Klein ${ }^{3}$, A. F. Jansson ${ }^{4}$, M. Hufnagel ${ }^{5}$, K. Minden ${ }^{1} .{ }^{1}$ German Rheumatism Research Center, Berlin, Germany; ${ }^{2}$ Vivantes Klinikum im Friedrichshain, Berlin, Germany; ${ }^{3}$ Asklepios Klinik St. Augustin, St. Augustin, Germany; ${ }^{4}$ Dr. von Hauner'sches Kinderspital, München, Germany; ${ }^{5}$ University of Freiburg, Pediatrics, Freiburg, Germany

Background: Systemic lupus erythematosus (SLE) is a clinically heterogeneous disease, which begins in childhood and adolescence in $15-20 \%$ of cases. Since 2004, data on SLE have been collected by means of a disease-specific questionnaire as part of the National pediatric rheumatology database (NPRD) in Germany. Since 2014, kidney biopsy results have been recorded to further specify kidney involvement.

Objectives: Evaluation of clinical signs and symptoms, outcome and laboratory data of patients with juvenile systemic lupus erythematosus from a large database in Germany.

Methods: Data from patients with SLE recorded in the NPRD in 2017 were considered for the analysis. In addition to age, sex, onset of disease, the criteria that led to the diagnosis, various laboratory parameters, organ involvement (current, ever) and therapy (current, last 12 months), current disease activity (numerical rating scale 0-10, NRS) and ECLAM (score 0-10) were recorded. Patient-reported outcomes included global assessments of overall-wellbeing and fatigue (NRS 0-10) and functional ability (CHAQ).

Results: 196 patients ( $86 \%$ female) with a median age of 16 years were documented. Criteria most frequently met at diagnosis included "antinuclear antibodies" $(88 \%)$, followed by "anti-ds-DNA-Ab" (66\%), "butterfly erythema" $(42 \%)$ and "arthritis" (41\%). A positive family history was found in $10 \%$ of patients.

At documentation, $85 \%$ of patients received disease-modifying anti-rheumatic drugs, most frequently hydroxychloroquine $(73 \%)$, followed by mycophenolate mofetil (32\%) and azathioprine (17\%). Systemic glucocorticoids obtained $52 \%$ of patients, $12 \% \geq 0.2 \mathrm{mg} / \mathrm{kg} /$ day. Biologics (rituximab $2 \%$ ) and cyclophosphamide i.v. $(3 \%)$ were rarely administered during the last 12 months. Disease activity was reported as 1.0 (NRS, median, IQR 0 - 9), ECLAM as 1.0 (median, range 0 - 10) In the laboratory, leukopenia $<3500 / \mu$ l was found in $9 \%$ of patients, lymphopenia $<1500 / \mu \mathrm{l}$ in $47 \%$ and erythrocyte sedimentation rate (ESR) $>25 \mathrm{~mm}$ in $15 \%$ of patients. Mean CHAQ was 0.24 , and $86 \%$ of patients had a $\mathrm{CHAQ}$ score $<0.5$. Mean patient's global assessment of overall-wellbeing was 1.5 , while the mean fatigue score was 2.86 (18\% NRS score $7-10)$.

The following organ involvement was ever present: general symptoms $84 \%$ skin/mucosa $72 \%$, joints $73 \%$, thyroid $15 \%$, muscle $25 \%$, lungs $17 \%$ and CNS $30 \%$. In 45/190 (24\%) patients, a kidney involvement was stated. In 34 patients $(75 \%)$ a kidney biopsy was performed and histology yielded the following results: Class 1: $6.7 \%$, Class 2: $16.7 \%$, Class 3: $40.0 \%$, Class $4: 23.3 \%$, Class 5: $13.3 \%$

Conclusion: The most common clinical symptoms documented in juvenile SLE patients were skin and joint involvement. In the course of the disease, a quarter of the patients developed kidney involvement, mostly proliferative nephritis. Apparently, azathioprine is increasingly being replaced by mycophenolate mofetil, biologicals have hardly been used so far. Although functional outcome and overall-wellbeing of jSLE patients was good, fatigue was a concern for some patients.

Disclosure of Interests: Claudia Sengler: None declared, Martina Niewerth: None declared, Nils Geisemeyer: None declared, Hermann Girschick: None declared, Ariane Klein Consultant of: Celgene, Annette Friederike Jansson: None declared, Markus Hufnagel: None declared, Kirsten Minden Consultant of: GlaxoSmithKline, Sanofi, Speakers bureau: Roche DOI: 10.1136/annrheumdis-2020-eular.3494

\begin{tabular}{|l}
\hline THU0516 \\
FIFTEEN CASES OF 3 NLR FAMILY MEMBERS \\
(NLRP3, NLRP12 AND NLRC4) RELATED \\
INFLAMMASOMOPATHIES IN A SINGLE CENTER OF \\
CHINA
\end{tabular}

W. Wang ${ }^{1}$, Y. Zhou ${ }^{1}$, H. Song ${ }^{1} .{ }^{1}$ Peking Union Medical College Hospital, Chinese Academy of Medical Sciences, Department of Pediatrics, Beijing, China

Background: There are four members in NLR family, NLRP3, NLRC4, NLRP1, and NLRP12, the mutations of which can lead to autoinflammatory diseases, while little reports describe those diseases in Chinese population.

Objectives: To report several cases of NLR-related autoinflammatory diseases in our center and to compare the differences of the presentations of CAPS between Chinese and western patients.

Methods: This study was undertaken at Peking Union Medical College Hospital (PUMCH) between 2012 and 2019. Demographic data, clinical presentations and genetic results were collected.

Results: 15 patients had been diagnosed as NLR-related autoinflammatory diseases in our center, including 11 CAPS, 1 FCAS4 and 3 NLRP12-AD patients.

We found 10 NLRP3 mutations, 3 NLRP12 mutations and 1 NLRC4 mutation. There are 3 novel mutations: NLRP3 c.1311G $>$ T, NLRP3 c.1711G $>A$, and NLRC4 c.514G>A

The major symptoms of those diseases are similar, such as recurrent episodes of fever associated with rash. And some may suffer from arthritis/arthralgia, uveitis, sensorineural deafness, symptoms of central neural systems (CNS).

On the other hand, different inflammasomopathies have unique characteristics. Symptoms of FCAS1, the mildest CAPS disorder, including rash and fever with/ without arthritis/arthralgia, usually develop in the first year of life. The onset age of MWS is later ( $8 \mathrm{~m}$ to $5 \mathrm{y}$ ), and those patients were more likely to develop arthritis/arthralgia, eye involvement, hearing loss and symptoms of CNS. NOMID was the most severe type, and was presented with chronic urticarial-like rash shortly after birth, as well as severe CNS manifestations and musculoskeletal involvement. One of our NOMID patients had clubbing fingers, which was not reported before. The onset age of NLRP12-AD ranges from $6 \mathrm{~m}$ to $5 \mathrm{y}$ and the presentation is similar to MWS while the FCAS4 patient presented with rash and fever, like FCAS1. 